\title{
Inversion of the western Pacific subtropical high dynamic model and analysis of dynamic characteristics for its abnormality
}

\author{
M. Hong ${ }^{1}$, R. Zhang ${ }^{1}$, J. X. Li ${ }^{1}$, J. J. Ge ${ }^{2}$, and K. F. Liu ${ }^{1}$ \\ ${ }^{1}$ Key Laboratory of Ocean Environment, Institute of Meteorology and Oceanography, PLA University of Science and \\ Technology, Nanjing, China \\ ${ }^{2}$ Meteorology and Hydrology Center of Nanjing M.C, Nanjing, China \\ Correspondence to: M. Hong (flowerrainhm@126.com)
}

Received: 4 December 2011 - Revised: 15 October 2012 - Accepted: 20 January 2013 - Published: 18 February 2013

\begin{abstract}
Based on time series data of $500 \mathrm{hPa}$ potential field from NCEP/NCAR (National Center for Environmental Forecast of American/National Center for Atmospheric Research), a novel consideration of empirical orthogonal function (EOF) time-space separation and dynamic system reconstruction for time series is introduced. This method consists of two parts: first, the dynamical model inversion and model parameter optimization are carried out on the EOF time coefficient series using the genetic algorithm (GA), and, second, a nonlinear dynamic model representing the subtropical high (SH) activity and its abnormality is established. The SH activity and its abnormal mechanism is studied using the developed dynamical model. Results show that the configuration and diversification of the $\mathrm{SH}$ equilibriums have good correspondence with the actual short-medium term abnormal activity of the SH. Change of SH potential field brought by the combination of equilibriums is more complex than that by mutation, and their exhibition patterns are different. The mutation behavior from high-value to low-value equilibriums of the SH in summer corresponds with the southward drop of the SH in the observed weather process. The combination behavior of the two steady equilibriums corresponds with disappearance of the "double-ridge" phenomenon of the SH. Dynamical mechanisms of these phenomena are explained.
\end{abstract}

\section{Introduction}

The western Pacific subtropical high is an important member of the East Asian summer monsoon systems. It has the interaction and feedback with the monsoon circulation, and both coexist in a nonlinear system. The abnormal activity of the western Pacific subtropical leads to frequent floods or droughts in areas near the Yangtze and Yellow rivers in China. For example, the super flood in drainage area of the Yangtze River in 1998 was induced by southward drop of the subtropical high (SH). In addition, the droughts with extreme high temperature in Chongqing and eastern Sichuan in 2006 summer, and the rainstorm in the drainage area of the Yellow River in July 2007 were also closely related with the continuous north and west location of the intra-seasonal SH. Abnormity of the SH in 2010 brought 14 rainstorms in South China, as well as south of the Yangtze River from May to July. Thus, mutation of the SH and its abnormal back-forth movements are closely related with summer flood of East Asia. Since the abnormality and activity of the SH is very complicated (Cao et al., 2002), it is very difficult to construct an accurate dynamic model for $\mathrm{SH}$ with nonlinear differential equations.

Inversion of dynamical model of meteorology elements or weather system evolvement from time series of the actual observation data can provide a feasible way to describe the physical mechanisms of the complicated weather system activity and abnormality. For example, Zhang et al. (2006) were concerned with the questions of local convergence of errors and its calculation during the inversion; they introduced the genetic algorithm (GA) to improve seeking root efficiency of model parameters. The application study on the inversion of nonlinear dynamical forecast model of the SH index was carried out (Zhang et al., 2008). Based on empirical orthogonal functions (EOFs) and the idea of dynamical model inversion, we established time-space (time-frequency) model foundation and retrieved a nonlinear dynamical model for SH. 
Aiming at the investigation of the summer SH abnormal activity in 2003 and 2006, based on the observed data, this paper inverts the corresponding nonlinear dynamical model of the SH, and then analyzes the activity and mutation mechanisms of the inverted dynamic model of the SH, including the equilibrium and stability of the SH system, and the dynamic characteristic analysis of dynamical action, such as bifurcation, and mutation induced by outer forces.

\section{Data and method}

\subsection{Research data}

Data used in this study are daily $500 \mathrm{hPa}$ potential field from NCEP/NCAR. We used data from 1 May 2003 to 31 October 2003 as the second half year of 2003, and the same for 2006 . The study range is $0-90^{\circ} \mathrm{N}, 90-180^{\circ} \mathrm{E}$ in the northeast hemisphere.

\subsection{EOF decomposition}

With EOF time (coefficients)-ispace (structure) separation method, the potential field series mentioned above can be time-space decomposed. The 2003-yr data are used as an example to be EOF-decomposed. From this analysis we find that the variance contributions of the first three character modes converge faster, and the cumulated variance contribution could reach $74.85 \%$ of the original field. The first mode reaches $65.16 \%$, which can basically describe the main characteristics of the potential field. After that, variance contribution reduces rapidly. Therefore, these first three EOF decomposition modes can be chosen as the modeling objects. Temporal and spatial characteristics of the first three modes of EOF decomposition are shown in Fig. 1.

\section{GA inversion of a nonlinear model of the SH}

\subsection{Basic idea for reconstructing the dynamical model}

Suppose that the physical time evolution law of a nonlinear system can be expressed as the following:

$$
\frac{d q_{i}}{d t}=f_{i}\left(q_{1}, q_{2}, \ldots, q_{i}, \ldots, q_{N}\right) \quad i=1,2, \ldots \ldots . N,
$$

where $f_{i}$ is the generalized nonlinear function of $q_{1}, q_{2}, \ldots, q_{i}, \ldots, q_{N} . N$ is the number of the state variables, and it can be generally determined according to the complexity of the dynamical system, which can be estimated by calculating its fractal dimension, $d$. If $N \geq 2 d+1$, the geometric structure of the dynamical system can be fully open. So $N \geq 2 d+1$ is a necessary but not sufficient condition. There are generally three methods with which $N$ can be derived from $d$ : the test algorithm, the false neighbor algorithm, and the improvement of the false neighbor algorithm (Lu and Zhang, 2010; Gouesbet, 1991). The difference form of Eq. (1) can be written as

$$
\begin{gathered}
\frac{q_{i}^{(j+1) \Delta t}-q_{i}^{(j-1) \Delta t}}{2 \Delta t}= \\
f_{i}\left(q_{1}^{j \Delta t}, q_{2}^{j \Delta t}, \ldots, q_{i}^{j \Delta t}, \ldots, q_{N}^{j \Delta t}\right) j=2,3, \ldots \ldots . M-1
\end{gathered}
$$

where $M$ is the time series length of observation data. Model parameters and system structure can be calculated from the observation data through inversion. $f_{i}\left(q_{1}^{j \Delta t}, q_{2}^{j \Delta t}, \ldots, q_{i}^{j \Delta t}, \ldots, q_{N}^{j \Delta t}\right)$ is an unknown nonlinear function that we suppose has $G_{j k}$ numbers of expanding items, including variable $q_{i}$ and $P_{i_{k}}$ numbers of corresponding function parameters $(i=1,2, \ldots N, j=1,2, \ldots M, k=$ $1,2, \ldots, K)$. It can be then supposed that $f_{i}\left(q_{1}, q_{2}, \ldots, q_{n}\right)=$ $\sum_{k=1}^{K} G_{j k} P_{i_{k}}$ (3). The matrix form of Eq. (2) is $\mathbf{D}=\mathbf{G P}$, where

$$
\begin{array}{r}
\mathbf{D}=\left\{\begin{array}{c}
d_{1} \\
d_{2} \\
\ldots \\
d_{M}
\end{array}\right\}=\left\{\begin{array}{c}
\frac{q_{i}^{3 \Delta t}-q_{i}^{\Delta t}}{q_{i}^{4 \Delta t}-q_{i}^{2 \Delta t}} \\
2 \Delta t \\
\ldots \\
\frac{q_{i}^{M \Delta t}-q_{i}^{(M-2) \Delta t}}{2 \Delta t}
\end{array}\right\}, \\
\mathbf{G}=\left\{\begin{array}{c}
G_{11}, G_{12}, \ldots \ldots G_{1 K} \\
G_{21}, G_{22}, \ldots \ldots G_{2, K} \\
\ldots \\
G_{M 1}, G_{M 2}, \ldots . G_{M, K}
\end{array}\right\}, \mathbf{P}=\left\{\begin{array}{c}
P_{i_{1}} \\
P_{i_{2}} \\
\ldots \\
P_{i_{K}}
\end{array}\right\} .
\end{array}
$$

Coefficients of the above generalized unknown equation can be identified through inverting the actual observation data. Given $\mathbf{D}$ and $\mathbf{P}$ can be solved to satisfy the above equation. If $q$ is considered as an unknown variable, Eq. (3) is a nonlinear system; but from another point of view, if $P$ is considered as unknown variable, Eq. (3) is a linear system. Classical least squares estimation can be used to make residual sum of squares $S=(\mathbf{D}-\mathbf{G P})^{T}(\mathbf{D}-\mathbf{G P})$ minimal, and then the regular equation $\mathbf{G}^{T} \mathbf{G P}=\mathbf{G}^{T} \mathbf{D}$ can be obtained.

$\mathbf{G}^{T} \mathbf{G}$ is usually a singular matrix, so we can easily solve it for its eigenvalues and eigenvectors. Through removing the points of "zero" values, the remaining components of $L$ numbers of $U_{1}, U_{2}, \ldots, U_{i}(i=1,2 \ldots, L)$ can form the diagonal matrix $\mathbf{U}$. With $V_{i}=\frac{G U_{i}}{\lambda_{i}}$, the matrix $\mathbf{V}=\left(V_{1}, V_{2}, \ldots V_{L}\right)^{T}$, and $\mathbf{H}=U \Delta^{-1} \mathbf{V}^{T}$ the equation $P=\mathbf{H D}$ is solved, and the parameter $P$ can be obtained.

Based on the above approach, the coefficients of the nonlinear dynamical systems can be determined. Therefore, the nonlinear dynamical equations of the corresponding observation data sequence can be obtained.

\subsection{Dynamical model inversion of the EOF time coefficients of potential field based on the search of GA}

Most of the parameter estimation methods currently used (such as neighborhood search method and least-squares 


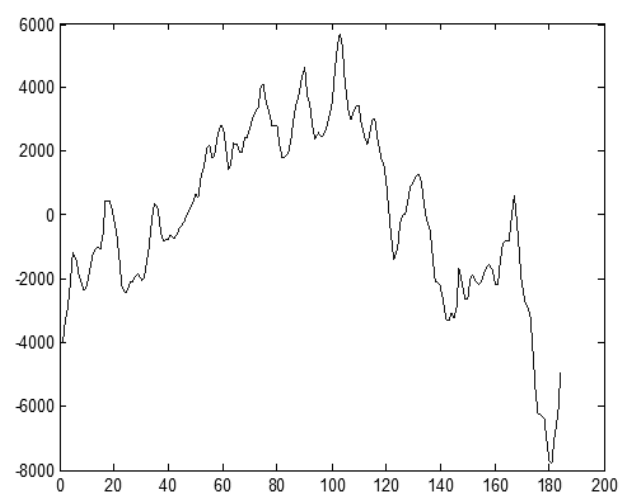

(a) The time series of the first mode

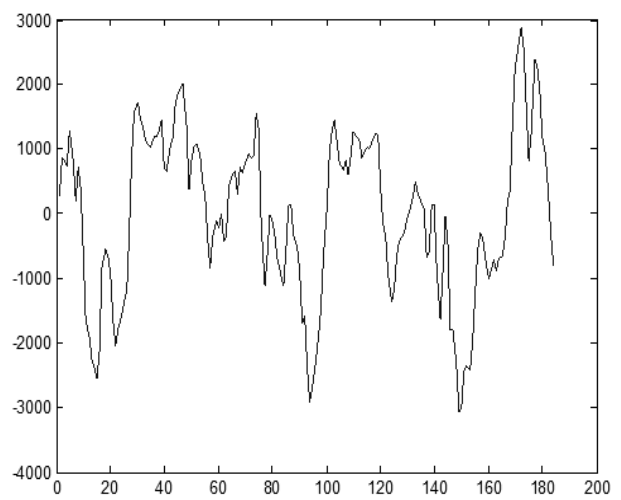

(c) The time series of the second mode

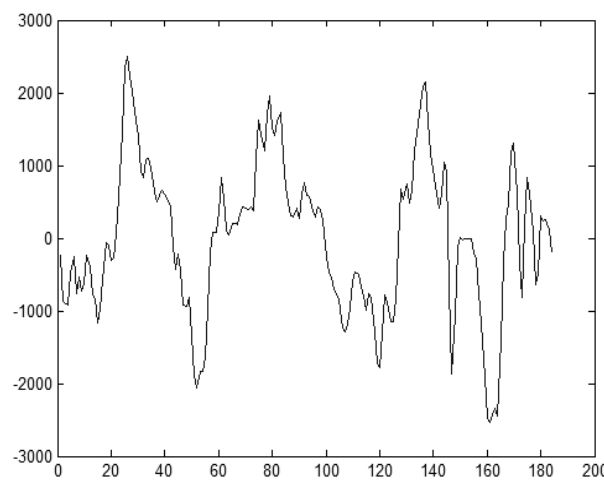

(e) The time series of the third mode

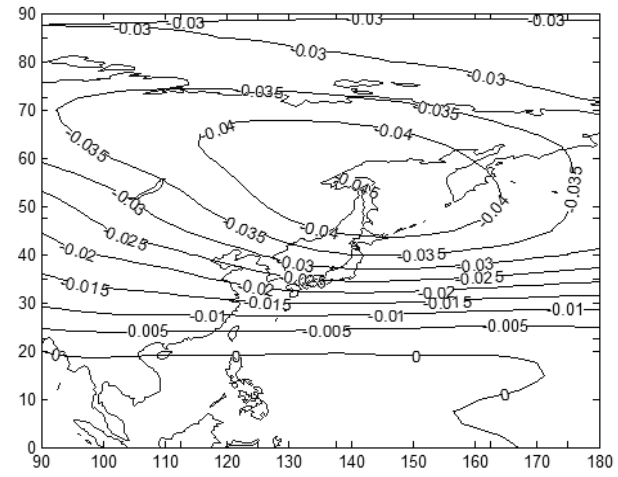

(b) The spatial mode of the first mode

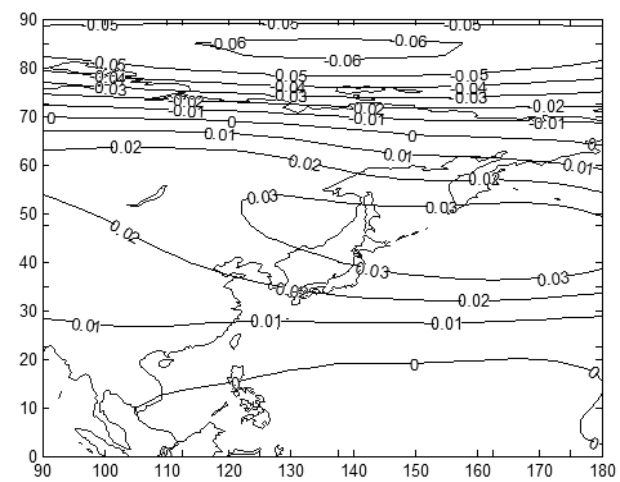

(d) The spatial mode of the second mode

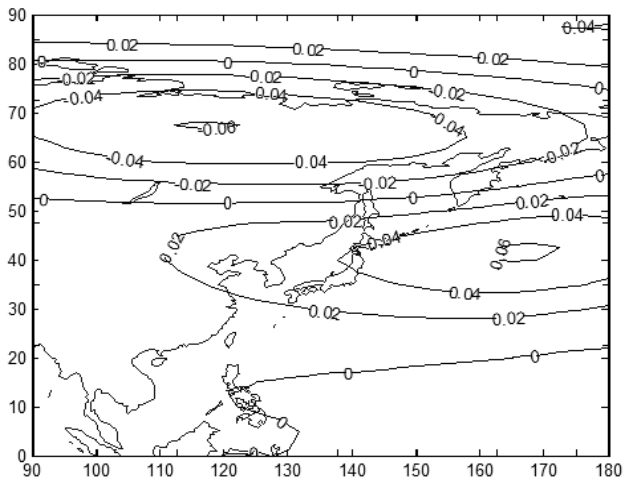

(f) The spatial mode of the third mode

Fig. 1. The time series and the spatial mode of the first three modes in 2003.

estimation) in the parameter space are one-way sequential search, which needs to explore the entire parameter space. Thus, the efficiency of the traditional method is very low. Because of the limitation of convergence of error gradient and its dependence on the initial solution, parameter estimation is prone to fall into a local optimum, rather than the global optimum (Wang, 2001). As a better alternative, we will introduce the GA to get the optimization and inversion of the mode parameters. GA has been developed in recent years, and it is extensively used as a global optimization algorithm. Its main features are global search and parallel computing, so it has very good parameter optimizing ability and quick error convergence rate.

The model parameter inversion is based on the basic principle expressed in Sect. 3.1, with a minimum square error $S=(\mathbf{D}-\mathbf{G P})^{T}(\mathbf{D}-\mathbf{G P})$ as the bound, using population (solutions) and parallel mode for parameters optimization in the parameter space search. Suppose that the 
following second-order nonlinear ordinary differential equations are taken as the dynamic model for inversion and reconstruction:

$$
\begin{aligned}
& \frac{\mathrm{d} X}{\mathrm{~d} t}=a_{1} X+a_{2} Y+a_{3} Z+a_{4} X^{2} \\
& +a_{5} Y^{2}+a_{6} Z^{2}+a_{7} X Y+a_{8} X Z+a_{9} Y Z \\
& \frac{\mathrm{d} Y}{\mathrm{~d} t}=b_{1} X+b_{2} Y+b_{3} Z+b_{4} X^{2} \\
& +b_{5} Y^{2}+b_{6} Z^{2}+b_{7} X Y+b_{8} X Z+b_{9} Y Z \\
& \frac{\mathrm{d} Z}{\mathrm{~d} t}=c_{1} X+c_{2} Y+c_{3} Z+c_{4} X^{2}+ \\
& c_{5} Y^{2}+c_{6} Z^{2}+c_{7} X Y+c_{8} X Z+c_{9} Y Z .
\end{aligned}
$$

$X, Y$, and $Z$ represent the time series of the first, second and third EOF modes, respectively. Based on the above reconstruction theory of the GA dynamical system and the actual observed data of 184 days from 1 May 2003 to 31 October 2003, parameters of the nonlinear dynamic model of the SH activity are inverted from the above three time-coefficient series. By computing and comparing the comparative variance contribution of each item in the mode, we synthetically analyze and eliminate the illusive item of the weak effect to the model in order to get the nonlinear dynamic model equations as the following, which can describe the SH activity in 2003:

$$
\left\{\begin{aligned}
\frac{\mathrm{d} X}{\mathrm{~d} t} & =-0.0801 Y-0.1607 Z-6.1803 \times 10^{-5} Y^{2} \\
& -3.4185 \times 10^{-5} Z^{2}+4.8721 \times 10^{-5} X Y \\
& +9.8524 \times 10^{-5} Y Z \\
\frac{\mathrm{d} Y}{\mathrm{~d} t} & =0.0943 X+0.2878 Z+6.5163 \times 10^{-5} Y^{2} \\
& -2.1517 \times 10^{-4} X Z+2.3394 \times 10^{-4} Y Z \\
\frac{\mathrm{d} Z}{\mathrm{~d} t} & =0.0207 X-0.2178 Y+1 \times 10^{-5} X^{2} \\
& -6.2465 \times 10^{-5} Y^{2}+2.3011 \times 10^{-5} X Y .
\end{aligned}\right.
$$

To test the accuracy and rationality of the above model, we need to carry out practical tests. Integral forecast experiments are carried out on the two inversion models. By setting the actual forecast initial value (selected from the index series), numerical fitting integration is taken on the time difference values of the models. The correlation coefficients between the fitting value and the actual value of the three time series differences are $0.7104,0.6509$ and 0.5632 , respectively. It means that the above inversion model can basically approach the actual potential field.

Similarly, the nonlinear dynamic model equations of the SH activity in 2006 can be obtained:

$$
\left\{\begin{array}{l}
\frac{d X}{d t}=-0.0831 Y-0.3604 Z+2.0885 \times 10^{-5} X^{2} \\
\frac{d Y}{d t}=-0.1337 Z+2.9344 \times 10^{-5} Y^{2} \\
+2.3401 \times 10^{-4} X Z+4.7833 \times 10^{-5} Y Z \\
\frac{d Z}{d t}=0.1189 X-0.1326 Y+3.2460 \times 10^{-5} X^{2} \\
-9.9232 \times 10^{-5} Z^{2}-1.0088 \times 10^{-4} X Y \\
-1.0770 \times 10^{-4} X Z-5.6600 \times 10^{-5} Y Z .
\end{array}\right.
$$

The correlation coefficients between fitting value and actual value of the three 2006 time series differences are 0.7606 , $0.7619,0.6595$, respectively. Compared with the dynamical model of the SH in 2003, the degree of the year 2006 (14 parameters) is slightly less complex than that of 2003 (16 parameters), and the forms of the equations and index items are different.

\section{Dynamical analysis of the SH activity}

\subsection{Discrimination theory of the equilibrium stability}

The singular spots (equilibrium state) of the dynamic system are a set of roots of the dynamic system equations, and they rarely change with time. Stability of the singular spots corresponds with stability of the dynamic system, and the nearby trajectory determines the character of singular spots. Usual singular spots can be classified into four types: crunodes, saddle-point, focus and center-point (Liu, 1965; Hassard et al., 1982). Taking model equations of the potential field as an example, we calculate the singular spot and discuss stability of the equilibrium depending on character of the eigenvalue of derivative-operator matrix. The nonlinear dynamic model Eqs. (5) and (6) of the potential field have generalized forms as Eq. (4).

As for large-scale weather systems, such as potential fields, when they are in a relatively stable state, the timevarying items of the dynamical model have small values and the left items of the equations can be seen as zero. So the singular spot can be achieved by solving the roots of equilibrium equations of the constant differential coefficient system. Then the stability can be analyzed.

The following formula (7) can be called derivativeoperator matrix:

$$
\left(\begin{array}{l}
\alpha_{11}=\left(\frac{\partial f}{\partial x}\right)_{x_{0}, y_{0}, z_{0}} \alpha_{12}=\left(\frac{\partial f}{\partial y}\right)_{x_{0}, y_{0}, z_{0}} \alpha_{13}=\left(\frac{\partial f}{\partial z}\right)_{x_{0}, y_{0}, z_{0}} \\
\alpha_{21}=\left(\frac{\partial g}{\partial x}\right)_{x_{0}, y_{0}, z_{0}} \alpha_{22}=\left(\frac{\partial g}{\partial y}\right)_{x_{0}, y_{0}, z_{0}} \alpha_{23}=\left(\frac{\partial g}{\partial z}\right)_{x_{0}, y_{0}, z_{0}} \\
\alpha_{31}=\left(\frac{\partial h}{\partial x}\right)_{x_{0}, y_{0}, z_{0}} \alpha_{32}=\left(\frac{\partial h}{\partial y}\right)_{x_{0}, y_{0}, z_{0}} \alpha_{33}=\left(\frac{\partial h}{\partial z}\right)_{x_{0}, y_{0}, z_{0}}
\end{array}\right) .
$$

The eigenvalue $\lambda$ of the derivative-operator matrix of the equations satisfies the following formula:

$$
\left[\begin{array}{ccc}
\alpha_{11}-\lambda & \alpha_{12} & \alpha_{13} \\
\alpha_{21} & \alpha_{22}-\lambda & \alpha_{23} \\
\alpha_{31} & \alpha_{32} & \alpha_{33}-\lambda
\end{array}\right]=0 .
$$

Equation of the characteristic roots can be converted as follows:

$$
\begin{aligned}
& \left(\alpha_{11}-\lambda\right)\left(\alpha_{22}-\lambda\right)\left(\alpha_{33}-\lambda\right)+\alpha_{13} \alpha_{21} \alpha_{32}+\alpha_{12} \alpha_{23} \alpha_{31} \\
& -\alpha_{13} \alpha_{31}\left(\alpha_{22}-\lambda\right)-\alpha_{23} \alpha_{32}\left(\alpha_{11}-\lambda\right) \\
& -\alpha_{12} \alpha_{21}\left(\alpha_{33}-\lambda\right)=0 .
\end{aligned}
$$


Table 1. The equilibrium roots of the summer SH equations in 2003.

\begin{tabular}{llll}
\hline The roots & $X$ & $Y$ & $Z$ \\
\hline First root & 1446.90 & 253.98 & -3921.69 \\
Second root & 4897.23 & 1642.73 & 1670.33 \\
Third root & $-1615.98-2720.07 \mathrm{i}$ & $-271.19+219.78 \mathrm{i}$ & $347.26+75.32 \mathrm{i}$ \\
Forth root & $-754.08+4658.23 \mathrm{i}$ & $-2907.56+2228.50 \mathrm{i}$ & $-559.24-592.37 \mathrm{i}$ \\
Fifth root & -5768.87 & -6165.60 & -22310.78 \\
Sixth root & $-754.08-4658.23 \mathrm{i}$ & $-2907.56-2228.50 \mathrm{i}$ & $-559.24+592.37 \mathrm{i}$ \\
Seventh root & $-1615.98+2720.07 \mathrm{i}$ & $-271.19-219.78 \mathrm{i}$ & $347.26-75.32 \mathrm{i}$ \\
\hline
\end{tabular}

Table 2. The equilibrium roots of the summer SH equations in 2006.

\begin{tabular}{llll}
\hline The roots & $X$ & $Y$ & $Z$ \\
\hline First root & 2228.34 & 1026.83 & -51.05 \\
Second root & 4224.90 & -19992.52 & $-5642.42 \mathrm{i}$ \\
Third root & 12239.86 & -78488.41 & -26771.97 \\
Forth root & 35578.87 & 87805.65 & -53112.08 \\
Fifth root & 258911.05 & -11981028.32 & -6645895.09 \\
Sixth root & $-1170.42+42.85 \mathrm{i}$ & $800.03-21.43 \mathrm{i}$ & $105.12+0.87 \mathrm{i}$ \\
Seventh root & $-1170.42-42.85 \mathrm{i}$ & $800.03+21.43 \mathrm{i}$ & $105.12-0.87 \mathrm{i}$ \\
\hline
\end{tabular}

A third-order equation of $\lambda$ can be obtained as follows:

$$
\begin{gathered}
\lambda^{3}-\left(\alpha_{11}+\alpha_{22}+\alpha_{33}\right) \lambda^{2}+\left(\alpha_{11} \alpha_{22}+\alpha_{11} \alpha_{33}\right. \\
\left.+\alpha_{22} \alpha_{33}+\alpha_{13} \alpha_{31}+\alpha_{23} \alpha_{32}+\alpha_{12} \alpha_{21}\right) \lambda \\
-\left(\alpha_{11} \alpha_{22} \alpha_{33}+\alpha_{13} \alpha_{22} \alpha_{31}+\alpha_{11} \alpha_{23} \alpha_{32}\right. \\
\left.+\alpha_{12} \alpha_{21} \alpha_{33}-\alpha_{21} \alpha_{32} \alpha_{13}-\alpha_{12} \alpha_{21} \alpha_{33}\right)=0 .
\end{gathered}
$$

Finally solutions of this third-order equation can be found, i.e., all of the characteristic roots of $\mathrm{DF}\left(x^{*}\right)$. We can judge whether the equilibrium is steady at this time based on the following three theorems (Alexander et al., 2001; Wang and Carey, 2005):

Theorem 1: When all of the characteristic roots of $\operatorname{DF}\left(x^{*}\right)$ have negative real parts, the equilibrium of system (4) is asymptotically stable.

Theorem 2: When at least one of the characteristic roots of $\mathrm{DF}\left(x^{*}\right)$ has positive real parts, the equilibrium of system (4) is unstable under Lyapunov sense.

Theorem 3: When all of the characteristic roots of $\mathrm{DF}\left(x^{*}\right)$ have zero real parts, the stability of equilibrium in system (4) depends on higher-order items of the Taylor series.

The situation of theorem 3 is called critical situation, which is often encountered in conservative systems. Based on the above discussion, we can judge the stability of the equilibrium.

\subsection{Discrimination of the equilibrium stability of the $\mathrm{SH}$}

Based on the above discrimination theory of equilibrium stability, seven roots can be found (Table 1) by solving equations of the SH inverted from 2003 summer data.

According to the equilibrium theory, there are three equilibriums states, which are the first, second and fifth roots, respectively. The theory in Sect. 4.1 is used to judge their stability. When the second group of roots $(4897.23,1642.73$, 1670.33 ) is substituted into the equations, all of the characteristic roots of $\mathrm{DF}\left(x^{*}\right)$ have negative real parts, so the solution is gradually stable. While the others are substituted into the equations, at least one characteristic root of $\mathrm{DF}\left(x^{*}\right)$ has positive real part, so they are unstable.

Not all the potential fields corresponding to each equilibrium state are shown due to the limitation of paper length. We only show potential field reconstructed from the stable root. As for the two unstable roots, we select the fifth root $(-5768.87,-6165.60,-22310.78)$ as an example, and reconstruct the potential field. Then we compare it with the potential field of the stable equilibrium. The two potential fields are shown in Fig. 2.

The potential field of the stable equilibrium is basically identical with the actual potential field, including the $5900 \mathrm{gpm}$ isoline, which can represent the SH. The potential field of the unstable equilibrium is very different from the actual potential field, such as its lowest potential height with the value of $3500 \mathrm{gpm}$, which does not exist in reality. 


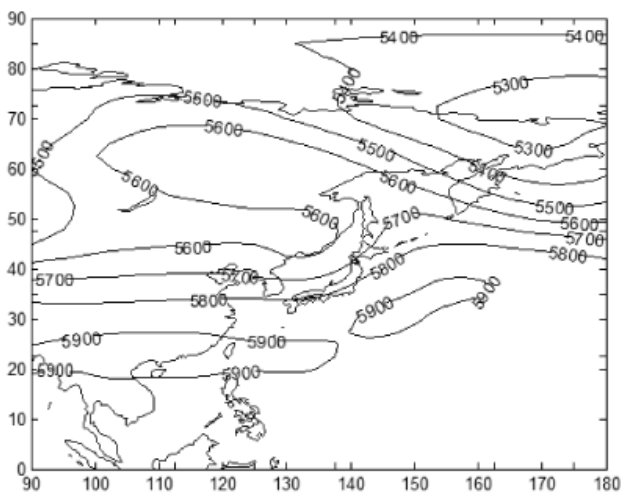

(a)

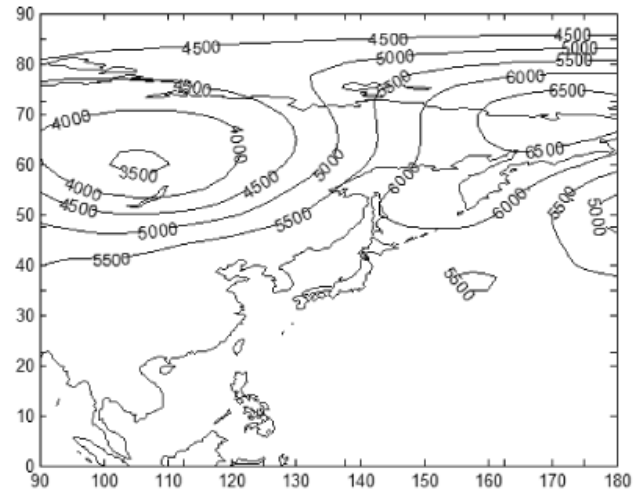

(b)

Fig. 2. (a) The potential field of the stable equilibrium and (b) the potential field of the unstable equilibrium of 2003.

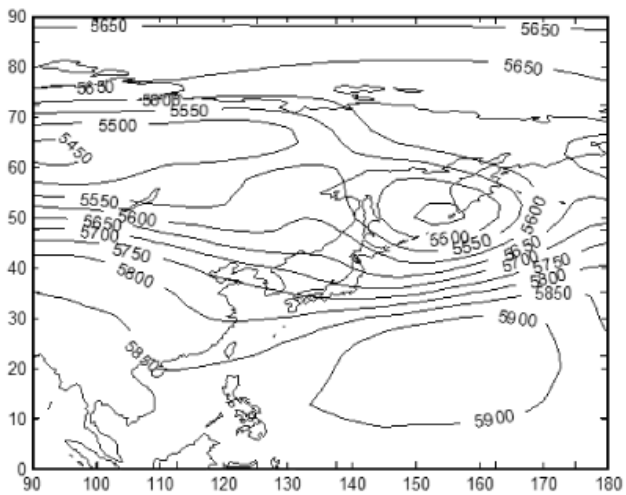

(a)

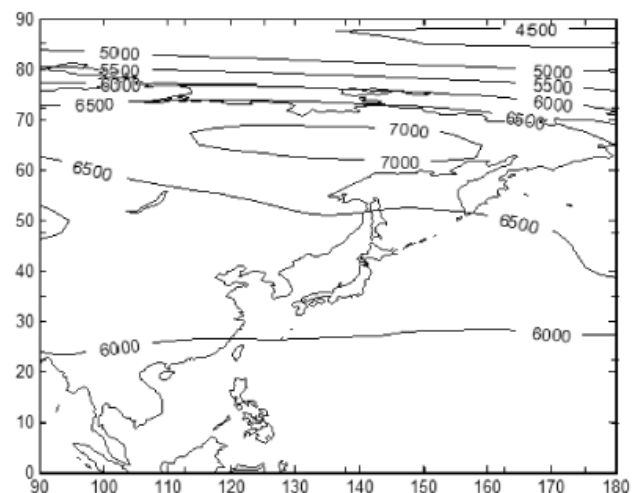

(b)

Fig. 3. (a) The potential field of the stable equilibrium and (b) the potential field of the unstable equilibrium of 2006.

Similarly, as for equations of the summer SH in 2006, the roots of equilibrium can be solved. Its seven roots are shown in Table 2.

There are five equilibriums among the seven roots, which are the first, second, third, fourth and fifth roots, respectively. Their numbers are more than that of the roots from 2003 data. Using the same theory applied before, their stability can be analyzed. The characteristic roots of $\operatorname{DF}\left(x^{*}\right)$ solved by substituting the first group of roots $(2228.34,1026.83,-51.05)$ to the equations all have negative real parts, so it is stable equilibrium. When the others are substituted into the equations, at least one characteristic root of $\operatorname{DF}\left(x^{*}\right)$ has positive real parts, so they are unstable.

Similarly to the 2003 data, we can draw potential field corresponding to each equilibrium. From Fig. 3, it can be seen that potential field of the stable equilibrium is basically identical with that of the actual potential field, while the potential field of unstable equilibrium has significant differences from the actual potential field, especially, the maximum at- mospheric pressure that can reach $7000 \mathrm{gpm}$, which is not possible in reality.

\subsection{Weather facts and variation characteristics of the SH activity}

The SH activity mainly behaves as seasonal variations and intraseasonal short-medium term variations. Seasonal variations of the SH location have much influence on the weather of China. There is much difference among seasonal variations of the SH in different years. Abnormal changes may appear in an individual year, which may lead to abnormal weather causing disasters such as droughts or floods in China (Li and Zhu, 2010; Qi et al., 2008). The short-medium term variation of the $\mathrm{SH}$ has a close relationship with the East Asian summer monsoon system (He et al., 2007). Using characteristic indexes of the SH defined by the Central Meteorological Observatory of China as reference, all of the characteristic indexes of summers (from May to October) from 


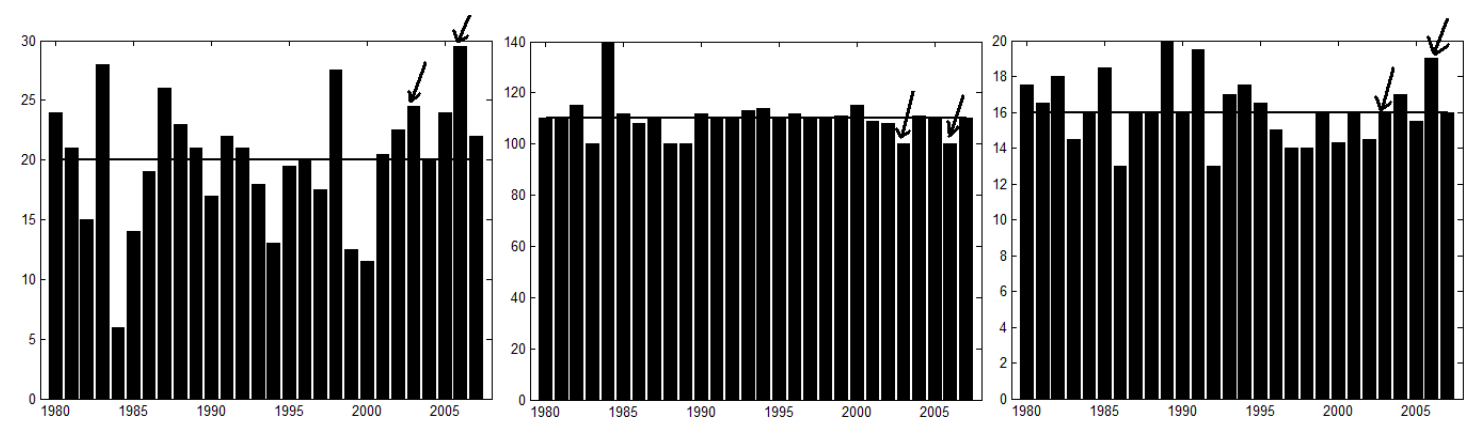

Fig. 4. The variation charts of the area index, the west ridge point index and the ridge line index of the SH in June from 1980 to 2007.
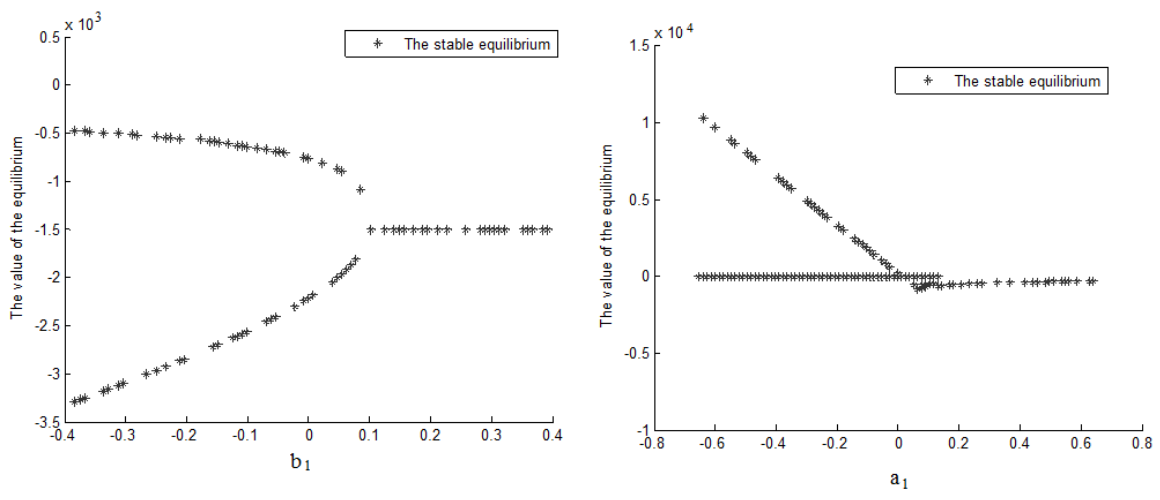

Fig. 5. (a) The first equilibrium (X) of the $\mathrm{SH}$ in 2003 varying with the outer parameters $b_{1}$ and (b) the first equilibrium (X) in 2006 varying with the outer parameters $a_{1}$.

years 1980 to 2007 (selecting June as an example) are calculated. Variation charts of the regional index, the west ridge point index and the ridge line index of different years are drawn (Fig. 4), in which straight lines represent average values of many years. It is shown in the charts that variations of indexes of the SH in different years are very different and some "abnormal" alterations appear in some years. The regional indexes of years 2003 and 2006 are both above the average value. Especially, the extreme value of the regional indexes of the $28 \mathrm{yr}$ appears in year 2006. Moreover, the west ridge point indexes of the two years are both under the average (shown as the arrowheads in Fig. $4 \mathrm{a}$ and b). All the above represent the wider range, the stronger intensity and the more western location of the $\mathrm{SH}$ of the two years among 28 years. On the other hand, the south and north locations of the SH of the two years are different: the ridge line of year 2003 is similar with the average, while that of year 2006 is obviously northern (shown as the arrowheads in Fig. 4c). All of the above facts show that the SH activities in the two years have both similar and different parts, while both are different from the average.

\subsection{Mechanisms of the dynamical character of the activity and aberrance of the $\mathrm{SH}$}

Based on the dynamical model of the SH derived from historical data, the discussion on the dynamical character and the explanation of the mechanism of the activity and aberrance of the SH can be developed. Setting the time variation of the left item of Eqs. (5) and (6) to zero, the dynamical equilibrium equations, which can represent moving back and forth activity of the summer SH in years 2003 and 2006, can be obtained. Using the means of numerical calculation, we calibrate one of the manipulative parameters to discuss the influence and the variation of equilibrium of the above equations, so that the evolution of the SH system with outer parameters can be drawn. When the stability of the equilibrium with outer parameters variation varies or the number and type of the equilibrium changes, the system is called in bifurcation. When the stable equilibrium jumps to another stable equilibrium with the outer parameter variation, the system is called in mutation.

Using the nonlinear dynamical model Eq. (5) of $500 \mathrm{hPa}$ SH activity inverted from 2003 data, and the nonlinear dynamical model Eq. (6) from 2006 data, the influence and variation of the SH activity and configuration with different model parameters are respectively discussed. Actually, 


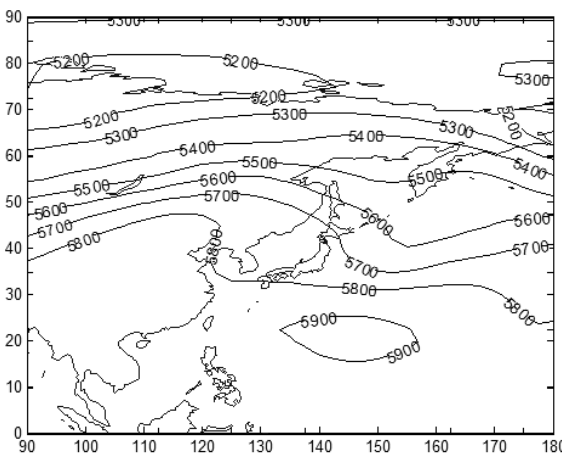

(a)

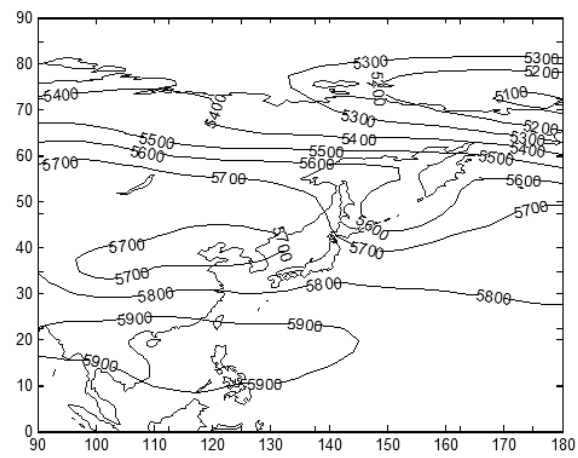

(b)

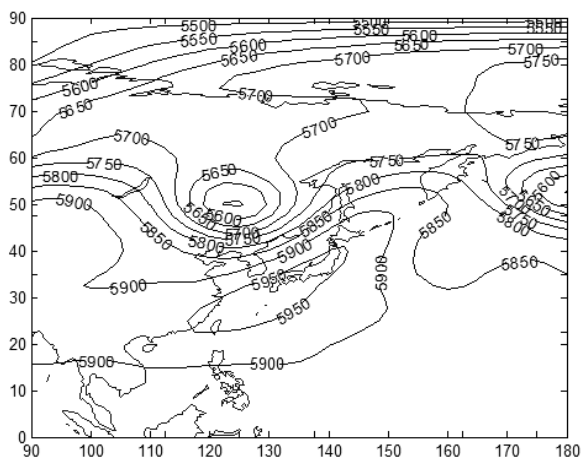

(c)

Fig. 6. The summer SH potential fields of (a) the high equilibrium and (b) the low equilibrium in 2003 reconstructed before combination with $b_{1}=-0.3$, and (c) after combination with $b_{1}=0.2$.

we estimate equilibrium stability of all of the 16 parameters in Eq. (5) and all of the 14 parameters in Eq. (6). After comparison, we find that the parameters $b_{1}, b_{9}, c_{7}$ and $a_{1}, b_{4}$, $b_{3}$ are the most representative of the combination, bifurcation and mutation. So representative parameters $b_{1}, b_{9}$, and $c_{7}$ for Eq. (5) are selected. As for Eq. (6), representative parameters $a_{1}, b_{4}$, and $b_{3}$ can be selected to carry out discussion. According to the above estimation of the equilibrium stability, it can be classified into three styles: combination, bifurcation and mutation. Due to the length limitation, only combination and bifurcation will be discussed in more detail in this paper. The evolution of the summer SH equilibrium with outer parameters in 2003 and 2006 will be calculated and discussed, respectively, and the corresponding dynamical mechanism discussion and weather fact explanation will be developed.

\subsubsection{Bifurcation and combination change of the equilibrium induced by outer parameters $b_{1}$ and $a_{1}$}

For the summer SH activity model of 2003 (Eq. 5), we select the outer parameter $b_{1}$; for the summer SH activity model of
2006 (Eq. 6), we select the outer parameter $a_{1}$. The equilibrium variations are shown in Fig. 5.

Because the variation of the three equilibriums is very similar, we just select the first equilibrium as an example. From Fig. 5, we can see that the variation trends of the equilibriums of the two models are similar. Equilibrium of the summer $\mathrm{SH}$ model in 2003 changes with $b_{1}$. When $b_{1}<0.1$, there are two equilibriums with very different numerical values, which are respectively high value equilibrium and low value equilibrium. With the increase of $b_{1}$, the difference between the two equilibriums reduces significantly. When $b_{1}>0.1$, the two equilibriums merge into a stable equilibrium. Then with the increase of $b_{1}$, the equilibrium is stable and rarely varies. The change of equilibrium of the summer model with $a_{1}$ in 2006 is very similar to above, and the only difference is the value of the equilibrium after combination, which is comparatively close to the value of one of the two equilibriums. In general, equilibriums of the two models can both represent the character of combination of two equilibriums, with the change of outer parameters.

Setting $b_{1}=-0.3$ and $b_{1}=0.2$, we represent the SH status before and after combination, respectively. Using the EOF time-space reconstruction of the corresponding $X, Y$, 


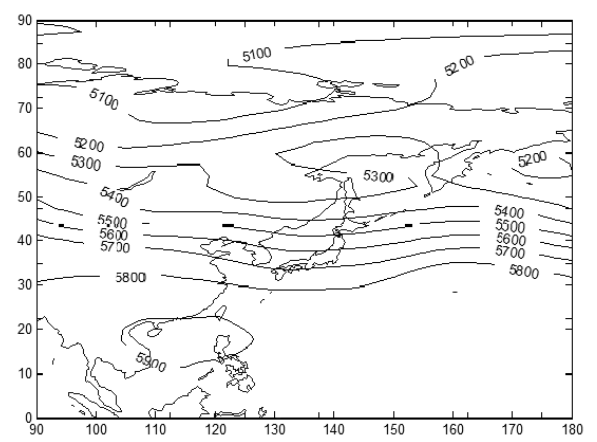

(a)

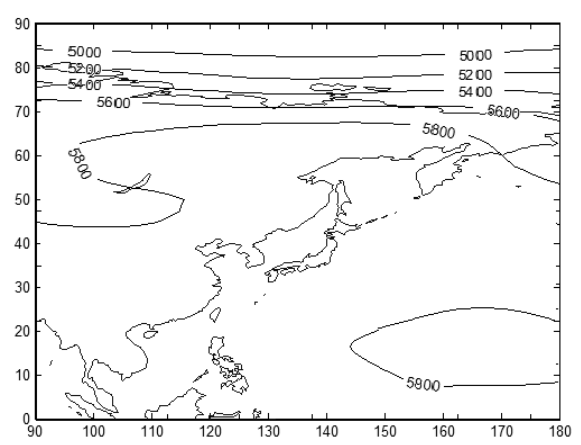

(b)

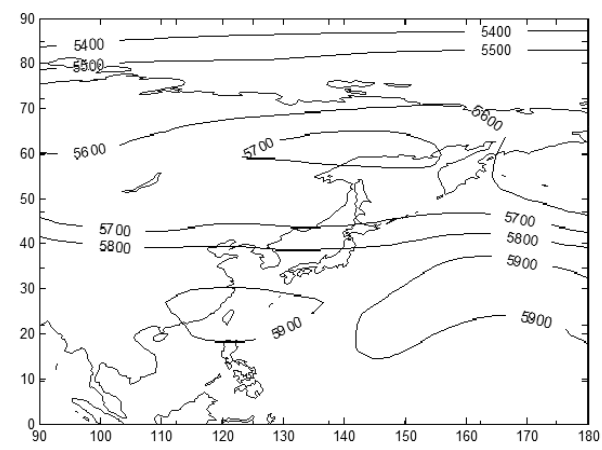

(c)

Fig. 7. The summer SH potential fields of (a) the high equilibrium and (b) the low equilibrium in 2006 reconstructed before combination with $a_{1}=-0.4$, and (c) after combination with $a_{1}=0.5$.
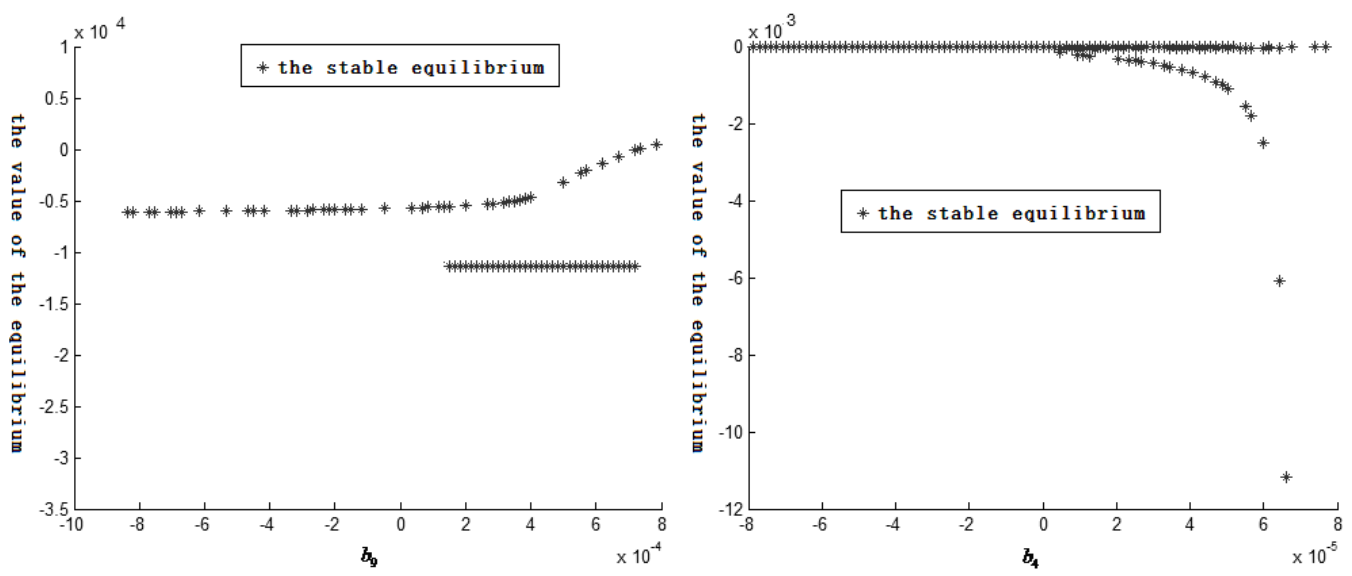

Fig. 8. (a) The first equilibrium (X) of the $\mathrm{SH}$ in 2003 varying with $b_{9}$ and (b) the first equilibrium (X) in 2006 varying with $b_{4}$.

and $Z$, we can get the summer SH potential fields in 2003 before and after combination, as shown in Fig. $6 \mathrm{a}, \mathrm{b}$ and c. Similarly, the summer SH potential fields in 2006 before combination with $a_{1}=-0.4$ and after combination with $a_{1}=0.5$ are shown in Fig. $7 \mathrm{a}, \mathrm{b}$ and c.

From Fig. 6, it can be seen that, as for the reconstructed summer SH potential field in 2003, before bifur- cation (bi-equilibrium state) the central area (5900 gpm isoline) of the $\mathrm{SH}$ is on the south side (central latitude is about $20^{\circ} \mathrm{N}$ ) and respectively is located in a west position (corresponding to low equilibrium) and east position (corresponding to high equilibrium). It can be seen as the circulation pattern of the South China Sea monsoon explosion or rainy season in South China from April to May, while 


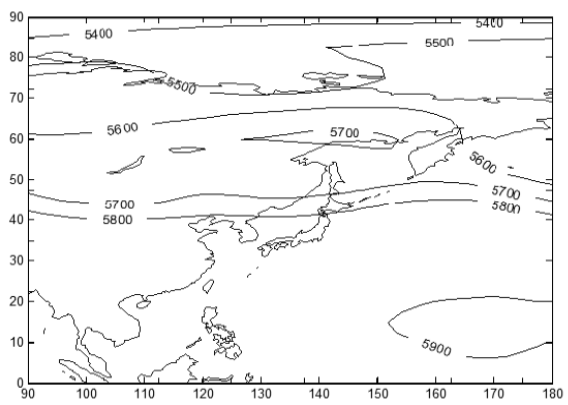

(a)

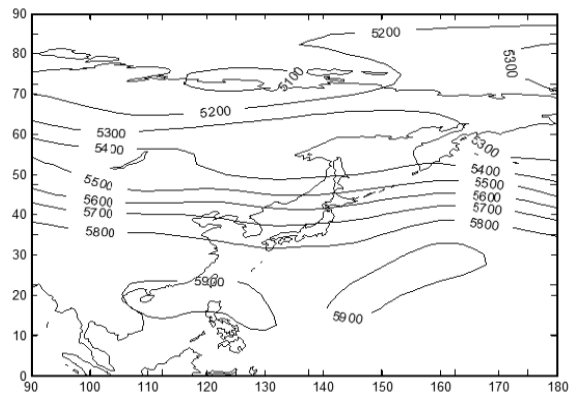

(b)

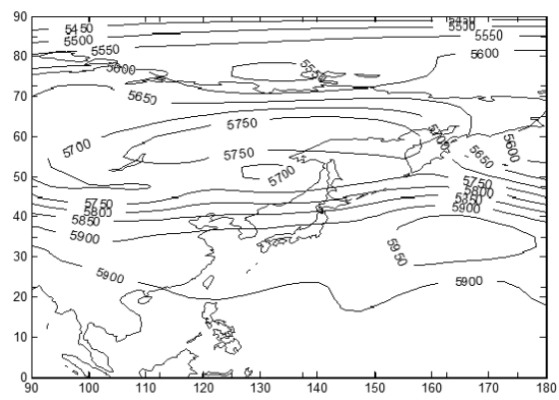

(c)

Fig. 9. The summer SH potential fields in 2003 reconstructed (a) before combination with $b_{9}=-4$, and (b) after combination of the high equilibrium and (c) the low equilibrium with $b_{9}=7$.

(a)

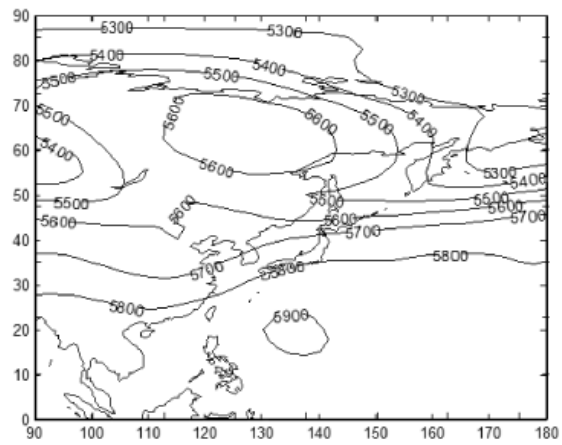

(b)
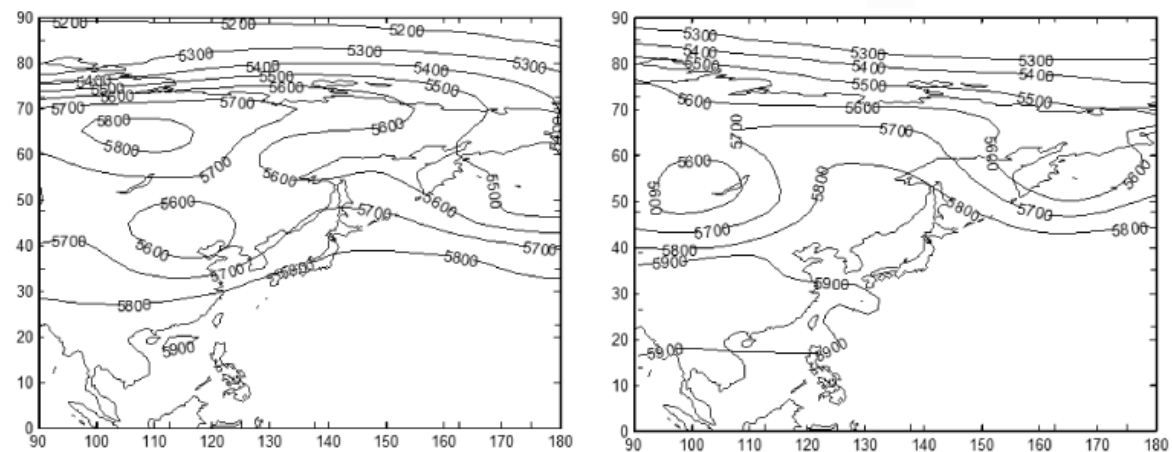

(c)

Fig. 10. The summer SH potential fields in 2006 reconstructed (a) before combination with $b_{4}=-4$, and (b) after combination of the high equilibrium and (c) the low equilibrium with $b_{4}=6$. 
after bifurcation (single-equilibrium state), the center area (5900 gpm isoline) of the $\mathrm{SH}$ goes north to $30^{\circ} \mathrm{N}$, performing the north leap course of the two west and east SH body at low latitudes (corresponding to the high and low equilibrium before bifurcation). It can be seen as the circulation form of the Yangtze and Yellow rivers in Meiyu period after north leap of the SH. So with the model parameter $b_{1}$ varying from -0.3 to 0.2 , there is an obvious north leap and enhancement course of the $\mathrm{SH}$, corresponding to $500 \mathrm{hPa}$ potential field (meaning the end of pre-rainy season of South China and the beginning of the Yangtze and Yellow rivers in Meiyu period). Then it maintains a stable equilibrium (corresponding to Meiyu circulation form).

From Fig. 7, we can see that the situation of 2006 is similar to 2003. Before bifurcation (bi-equilibrium state), the central area of the SH (5900 gpm isoline) is south of that in 2003 (the central latitude is about $15^{\circ} \mathrm{N}$ ), and is located in the west position (low equilibrium state) and east position (high equilibrium state), respectively. It is similar to the circulation form during the pre-rainy season of the South China from April to May. After bifurcation (single-equilibrium state), the central area (5900 gpm isoline) of the $\mathrm{SH}$ also goes north (the center is located at $25^{\circ} \mathrm{N}$ ), but the intensity changes less, and it does not merge. Location of the SH with weak intensity is south and west. So the SH north leap of $500 \mathrm{hPa}$ is less obvious than the one in 2003, with the model parameter $a_{1}$ from -0.4 to 0.5 . Then it maintains a south-west stable equilibrium (the west point of the $\mathrm{SH}$ goes west and maintains at $110^{\circ} \mathrm{E}$, which led to the continuous summer warm and dry weather in Sichuan and Chongqing areas of China in 2006).

\subsubsection{Bifurcation and discretization change of equilibrium induced by outer parameters $b_{9}$ and $b_{4}$}

With the change of outer parameters $b_{9}$ and $b_{4}$, variation of the equilibrium stability and aberrance change of the SH potential field are shown in Fig. 8, and they are put together to be discussed.

From Fig. 8, we can see that the changing trends of equilibriums of the two modes are similar. Equilibrium of the summer SH model changes with $b_{9}$. When $b_{9}<0.0001$, there is one equilibrium state. But when $b_{9}>0.0001$, the equilibrium state is divided into two equilibriums. With the increase of $b_{9}$, the low value equilibrium maintains stablity. The initial equilibrium of the summer SH in 2006 is stable with increase of $b_{4}$ after bifurcation, but the other equilibrium from bifurcation reduces quickly with the increase of $b_{4}$, showing a characteristic of quick change.

With EOF time-space reconstruction, the SH potential fields in 2003 before bifurcation with $b_{9}=-4$ and after bifurcation with $b_{9}=7$ can be obtained (Fig. $9 \mathrm{a}, \mathrm{b}$ and c). The summer SH potential fields in 2006 before bifurcation $b_{4}=-4$ and after bifurcation $b_{4}=6$ are shown in Fig. 10a,b and $\mathrm{c}$.
Dynamical model of the SH in 2003 (Fig. 9) shows a stable equilibrium state before bifurcation, and the corresponding range of the $\mathrm{SH}(5900 \mathrm{gpm})$ is located to the east $\left(150^{\circ} \mathrm{E}\right.$ east) of the Pacific Ocean in low latitude (center is $15^{\circ} \mathrm{N}$ ), which is similar to the circulation in early spring season. After bifurcation, there are two possible $\mathrm{SH}$ activity forms. One extends west to the South China area (some parts are still in the sea), and its north leap course is not evident (Fig. 9b). The other shows obvious enhancement and north leap of the $\mathrm{SH}$ (center of $5900 \mathrm{gpm}$ isoline still is located near $30^{\circ} \mathrm{N}$ and extends to the west of $100^{\circ} \mathrm{E}$ ), which is similar to the circulation form of the abnormal strong and northwest $\mathrm{SH}$ in the summer of 2003. The above results show that $b_{9}$ is an important force factor leading to abnormal enhancement and north leap of the SH.

Dynamical model of the summer SH in 2006 (Fig. 10) shows a stable equilibrium state before bifurcation, corresponding to a weak SH form (5900 gpm isoline). After bifurcation, there are two possible SH activity forms. One is similar to the form before bifurcation (still maintaining weak SH form) (Fig. 10b). The other shows obvious enhancement and north leap of the SH (the center of $5900 \mathrm{gpm}$ isoline located near $25^{\circ} \mathrm{N}$ ) and west extension (to the west of $100^{\circ} \mathrm{E}$ ), which is similar to the circulation form of the abnormal west SH in the summer of 2006, which remains stable (leading to continuous droughts in Sichuan and Chongqing areas). The above results show that $b_{4}$ is an important force factor leading to abnormal west extension of the $\mathrm{SH}$.

Similarly, change of outer parameters $c_{7}$ and $b_{3}$ will lead to the mutation change of equilibrium and the corresponding $\mathrm{SH}$ potential fields. But their degree of complexity is less than those of the above bifurcation and combination. This point is not discussed extensively in this paper due to the length limitation.

\section{Conclusions}

Due to the complexity of the East Asian summer monsoon system and summer SH activity, a dynamical model to describe SH activity has not established so far. In this study, a novel method has been put forward by which dynamical model of the SH potential field can be reconstructed from the observation data. Then a dynamical model of the SH is inverted and reconstructed using GA. After that, we investigated the mechanism of SH activity and mutation including the equilibrium and stability of the SH system, and analyzed the dynamical characteristic and dynamical action, such as bifurcation and mutation induced by the external forcing. Finally, the developed method is used to analyze the actual SH conformation and to depict the main weather characteristics.

Analytical results show that, with the advantages of the global optimization and parallel calculation by GA, the nonlinear dynamical model of the SH potential field can be reconstructed exactly and quickly, and the change of 
parameters in this model can lead to instability and bifurcation of the equilibrium of the $\mathrm{SH}$ system. Comparing the $\mathrm{SH}$ potential field models of the 2003 summer and the 2006 summer with their weather characteristics, we find that the distribution and variation of the equilibrium of the $\mathrm{SH}$ model have good correspondence with the actual short-medium term abnormal activity of the SH. Our analysis also shows that the change brought by equilibrium change of bifurcation and combination is more complex than that brought by mutation. In particular, the leap from high value equilibrium to the low in the summer SH system corresponds with the dynamical behavior of the SH southward drop. Combination of the two steady equilibriums corresponds with the disappearance of the "double-ridges" phenomenon of the SH.

Acknowledgements. This study is supported by the National Natural Science Foundation of China (41075045), the Young Scientists Fund of the National Natural Science Foundation of China (41005025/D0505), the National Natural Science Foundation (BK2011123) of Jiangsu Province and the Predicted Foundation of PLA University of Science and Technology. Helpful comments and suggestions from editors and two reviewers are highly appreciated.

Edited by: M. Fedi

Reviewed by: A. De Santis and one anonymous referee

\section{References}

Alexander, A., Nepomany, A., and Ilya, B. S.: Stability of Thermocapillary Flows with Inclined Temperature Gradien, J. Fluid. Mech., 442, 141-155, 2001.

Cao, J., Huang, R. H., Xie, Y. Q., and Tao, Y.: Evolution mechanism of the Western Pacific subtropical high, Sci. China Ser. D.-Earth Sci., 46, 659-666, 2002.
Gouesbet, G.: Reconstruction of the vector fields of continuous dynamical systems form numerical scalar time series, Phys. Rev. A, 43, 5321-5331, 1991.

Hassard, B. D., Kazarinoff, W. D., and Wan, Y. H.: Theory and Application of Hopf Bifurcation, Cambridge University Press, Cambridge, 1982.

He, J. H., Qi, L., Wei, J., and Chi, Y. Z.: Reinvestigations on the East Asian Subtropical Monsoon and Tropical Monsoon, Chinese J. Atmos. Sci., 6, 1257-1265, 2007.

Li, J. P. and Zhu, J. L.: Climatological features of the Western Pacific subtropical high southward retreat process in late-spring and early-summer, Acta Meteorologica Sinica, 4, 397-412, 2010.

Liu, S. Z.: N-dimensional topological singularities of classification, Adv. Math., 8, 217-242, 1965.

Lu, X. T. and Zhang, J. Q.: Nonlinear physics, Press of University of Science and Technology of China, Anhui, 2010.

Qi, L., Guan, Z. Y., Zhang, Z. Q., and He, J. H.: Climatological View on Double Ridges Process of the Western Pacific Subtropical High and Its Relation to Quasi-10-day Oscillation of Monsoon Trough, Chinese J. Atmos. Sci., 32, 165-174, 2008.

Wang, L.: Intelligent Optimization Algorithms and Its Application, Tsinghua University Press, Beijing, 2001.

Wang, X. W. and Carey, G. F.: On Marangoni Effects in a Heated Thin Fluid Layer with a Monolayer Surfactant. Part I: Model Development and Stability Analysis, Int. J. Numer. Meth. Fluids, 48, 1-16, 2005.

Zhang, R., Hong, M., Sun, Z. B., Niu, S. J., Zhu, W. J., Min, J. Z., and Wan, Q. L.: Non-linear Dynamic Model Retrieval of Subtropical High Based on Empirical Orthogonal Function And Genetic Algorithm, Appl. Math. Mech., 27, 1645-1654, 2006.

Zhang, R., Hong, M., Wang, H. Z., Chen, Y. D., and Wan, Y. L.: Retrieval the non-linear dynamic forecast model of El Nino/La Nina index based on the genetic algorithm optimization, Chinese J. Geophys., 51, 633-645, 2008. 